\title{
Der Einschluss von Frauen in klinische Arzneimittelstudien*
}

P. Kleist

Zur Untersuchung potentieller geschlechtsbezogener Unterschiede bezüglich Wirksamkeit und Sicherheit von Arzneimitteln ist ein ausreichender Einschluss von Frauen in klinische Studien erforderlich. Die bestehenden gesetzlichen Regelungen für die Entwicklung neuer Arzneimittel scheinen die adäquate Berücksichtigung von Frauen in zulassungsrelevanten Studien zu garantieren. Bei anderen Studien, die von diesen Bestimmungen nicht in gleicher Weise erfasst werden, ist die Situation nicht befriedigend. Dies betrifft insbesondere Forschungsprojekte aus dem akademischen Bereich (z.B. optimiertes Management einer Erkrankung oder Morbiditäts-/Mortalitätsstudien). Die forschende Industrie, Studienärzte und Ethikkommissionen sollten geschlechtsbezogene Ansätze bei der Planung und Auswertung bzw. bei der Begutachtung klinischer Arzneimittelstudien im Auge behalten.

\footnotetext{
* Ein ausführlicher Beitrag zur Thematik erschien in «Applied Clinical Trials» unter dem Titel: Women and Trials: When is Gender a Consideration? (ACT 2005;June:72-84).
}

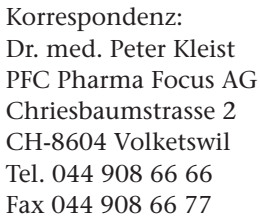

\section{Historische Entwicklung}

Nicht zuletzt aufgrund der Thalidomidtragödie wurde die klinische Arzneimittelforschung an Frauen lange Zeit als riskant eingestuft. Richtlinien der Arzneimittelbehörden, insbesondere in den USA, schlossen Frauen aus den frühen Entwicklungsphasen von Arzneimitteln aus [1]; Sicherheitsbedenken und die Furcht vor Haftungsfällen hielten Studienärzte generell davon ab, Frauen in klinische Studien einzuschliessen. Somit war bis Ende der 80er Jahre die Arzneimittelforschung praktisch eine Forschung an Männern, deren Ergebnisse für die Behandlung von Frauen extrapoliert wurden. Eine Abkehr von dieser rigiden Einstellung lässt sich auf zwei Entwicklungen zurückführen: einerseits auf die anwachsende Erkenntnis, dass geschlechtsbezogene Unterschiede der Pharmakokinetik und Pharmakodynamik klinisch bedeutsam sein könn(t)en; andererseits auf die zunehmende Fragwürdigkeit einer Verweigerung des Rechts von Frauen, an klinischen Studien teilzunehmen. Gestützt auf den «Belmont Report», der 1979 die Grundprinzipien einer ethischen biomedizinischen Forschung konstatierte [2], beriefen sich Patientinnen in den USA auf ihre autonome Entscheidungskompetenz und ihr Selbstbestimmungsrecht, vor allem in bezug auf die Teilnahme an Studien zu neuen Aidsmedikamenten. Als Folge eines Paradigmenwechsels schreibt die amerikanische Gesundheitsbehörde FDA seit 1993 den adäquaten Studieneinschluss von
Frauen zur Untersuchung potentieller geschlechtsbezogener Unterschiede in der Wirkung und Sicherheit von Arzneimitteln verbindlich vor [3]. Seit 2000 ist die FDA sogar gesetzlich bevollmächtigt, eine Studie oder eine ganze Arzneimittelentwicklung zu stoppen, falls ein Sponsor Frauen von der Untersuchung von Arzneimitteln zur Therapie lebensbedrohlicher Erkrankungen ausschliesst [4].

Da faktisch für jedes bedeutsame neue Arzneimittel der Zutritt zum amerikanischen Markt angestrebt wird, haben die US-Regelungen globale Auswirkungen. Inzwischen fordern auch die weltweit berücksichtigten Richtlinien der International Conference on Harmonisation den ausreichenden Einschluss von Frauen ab Phase 1 der Arzneimittelentwicklung und geschlechtsbezogene Studienauswertungen. Selbst in internationalen forschungsethischen Leitlinien, wie beispielsweise den CIOMS/WHO-Richtlinien, ist der Einschluss von Frauen in biomedizinische Forschungsprojekte als ethisches Prinzip beschrieben [5].

\section{Die heutige Situation}

Dass die regulatorischen Vorgaben im Rahmen der Entwicklung neuer Arzneimittel «greifen», haben unlängst fünf verschiedene Untersuchungen in den USA, Europa und Japan zur Berücksichtigung von Frauen in zulassungsrelevanten Arzneimittelstudien bestätigt: insgesamt ist der Anteil von Frauen und Männern heute nahezu vergleichbar [6]. Geringere Einschlussraten von Frauen in Phase-1-/frühe Phase-2-Studien sind insofern von untergeordneter Relevanz, als der Frauenanteil dennoch ausreichend erscheint, um frühzeitig auf geschlechtsbezogene Unterschiede aufmerksam zu werden und nachfolgende Studien auf rationaler Basis zu planen. In den Entwicklungsphasen 2 (Dosisfindung) und 3 (Nachweis von Wirksamkeit und Sicherheit an grossen Patientenkollektiven) beträgt der Frauenanteil im Mittel etwa 50\%. Aufgrund dieser Tatsache hat zum Beispiel die europäische Arzneimittelbehörde erst kürzlich davon abgesehen, eigene Richtlinien zur Berücksichtigung von 
Frauen in der klinischen Entwicklung von Arzneimitteln zu erlassen.

Allerdings decken alle diese Studien, deren oftmals alleiniges Ziel in der Dokumentation von Wirksamkeit und Sicherheit neuer medikamentöser Therapien besteht, nur einen Teil der durchgeführten klinischen Arzneimittelstudien ab. Studien zum optimalen Management einer Erkrankung oder grosse Morbiditäts-/Mortalitätsstudien sind immer noch eine Domäne der akademischen Forschung und werden von den arzneimittelzulassungsrelevanten Vorgaben der Behörden nicht in gleichem Umfang erfasst. Wie stellt sich die Situation in bezug auf diesen Bereich der klinischen Forschung dar?

Seit 1993 verlangt in den USA auch das National Institute of Health den adäquaten Einschluss von Frauen in alle Studien, die mit öffentlichen Geldern unterstützt werden [7]. Vorzugsweise betrifft diese Förderung die nichtkommerzielle, akademische Forschung. Jüngste Analysen haben jedoch gezeigt, dass die Situation - trotz der Abhängigkeit von finanzieller Förderung - nach wie vor unbefriedigend ist [8, 9]. Legt man die in den letzten Jahren durchgeführten repräsentativen Metaanalysen von publizierten klinischen Originalstudien zugrunde, deren Ziel in der Untersuchung der relativen Einschlussrate von Frauen bestand [8-14] und in denen schwerpunktmässig nichtkommerzielle Forschungsprojekte enthalten sind, lässt sich folgendes zusammenfassen: im Mittel beträgt der Frauenanteil nur 25\%. Geschlechtsspezifische Datenauswertungen finden sich nur in jeder dritten publizierten Studie. In 10-15\% der Studien wurden Frauen immer noch ausgeschlossen. Vor allem in grossen Mortalitätsstudien und Studien mit kardiovaskulären Fragestellungen sind Frauen unzureichend repräsentiert [11, 15]. Im Verhältnis zur Krankheitsprävalenz ist der Einschluss von Frauen mit Herzinsuffizienz besonders niedrig [13]. Es ist davon auszugehen, dass sich die Situation in der Schweiz nicht grundlegend anders darstellt.

\section{Klinische Relevanz}

Heute ist hinreichend bekannt: Geschlechtsbezogene Unterschiede in der Pharmakokinetik und Pharmakodynamik von Arzneimitteln sind meistens subtil und häufig ohne klinische Relevanz. Anpassungen der Dosis aufgrund unterschiedlicher Wirkungs- bzw. Nebenwirkungsprofile sind selten notwendig. Bei 185 Arzneimitteln, welche durch die FDA zwischen 1995 und 1999 zugelassen wurden, war in keinem Fall eine
Dosisanpassung für die Behandlung von Frauen notwendig (zusammenfassende Übersicht in [6]). Es scheint, dass geschlechtsbezogene anatomische und physiologische (z.B. Körperfettverteilung oder renale Clearance) sowie geschlechtsspezifische Unterschiede (Einfluss von Zyklus und Menopause) generell keine stärkeren Auswirkungen auf die Arzneimittelwirkung haben als die ohnehin bestehenden interindividuellen Unterschiede zwischen Patienten (z. B. bezüglich Körpergewicht). Und dennoch: an zwei Beispielen soll verdeutlicht werden, dass die Frage nach geschlechtsbezogenen Arzneimittelwirkungen, insbesondere im Kontext des Krankheitsmanagements, nicht unter den Tisch fallen darf. 1997 wurde die placebokontrollierte Digoxin-Studie an 6800 herzinsuffizienten Patienten durch die Digitalis Investigation Group publiziert [16]. Zwar war nur ein leichter Trend zur Mortalitätsreduktion unter Digoxingabe nachweisbar, die Hospitalisierungsrate in der Digoxingruppe war jedoch statistisch signifikant erniedrigt. Erst vier Jahre später wurde die Studie, deren Frauenanteil lediglich $22 \%$ betrug, von einer anderen Studiengruppe geschlechtsbezogen ausgewertet [17]: die Prognose digitalisierter Frauen war schlechter als unter Placebo, und die nur leicht erniedrigte Hospitalisierungsrate unter Digoxin erreichte - im Gegensatz zu den Ergebnissen bei Männern - keine statistische Signifikanz. Das zweite Beispiel betrifft die Verabreichung von Acetylsalicylsäure (ASS) zur kardiovaskulären Primärprävention. Kontrollierte Studien haben in der Vergangenheit die Wirksamkeit niedrig dosierter ASS zur Primärprävention des Myokardinfarkts bei Männern belegt, während das Schlaganfallrisiko praktisch nicht beeinflusst wurde. In einer zu Beginn des Jahres publizierten Studie [18], die dieser Frage an nahezu 40000 Frauen nachging, zeigte sich überraschenderweise ein ganz anderes Bild: das Risiko eines ischämischen Insults wurde bei Frauen signifikant verringert - das Infarktrisiko jedoch praktisch nicht.

\section{Schlussfolgerungen}

Die Bedeutung geschlechtsbezogener Unterschiede darf nicht überbewertet werden. Dennoch zeigen einzelne Beispiele, dass bestehende Unterschiede klinisch relevant sein können. Im Rahmen der Entwicklung neuer Arzneimittel besteht heute ein geschärftes Problembewusstsein, nicht zuletzt durch behördliche Regelungen, die eine adäquate Repräsentation von Frauen in klinischen Studien vor Erteilung der Marktzulassung fordern. Doch die zulassungsrelevanten 
Studien decken längst nicht alle wichtigen therapeutischen Fragestellungen ab. Sowohl die pharmazeutische Industrie als auch forschende Ärzte sollten bei der Planung neuer Studien dafür Sorge tragen, dass Patientinnen ausreichend Berücksichtigung finden und die Daten anschliessend geschlechtsbezogen analysiert werden. Eine regulative Funktion kommt auch den Ethikkommissionen zu. In Deutschland ist man bereits einen Schritt weitergegangen. Gemäss der deutschen GCP-Verordnung [19] ist dem Antrag zur Begutachtung einer Studie eine Begründung beizufügen, «dass die gewählte Geschlechterverteilung in der Gruppe der betroffenen Personen zur Feststellung möglicher geschlechtsspezifischer Unterschiede bei der Wirksamkeit oder Unbedenklichkeit des geprüften Arzneimittels angemessen ist $[\ldots]$...

\section{Literatur}

1 US Department of Health, Education, and Welfare. General Considerations for the Clinical Evaluation of Drugs. HEW (FDA) 77-3040. Washington: Government Printing Office; 1977.

2 National Commission for the Protection of Human Subjects of Biomedical and Behavioral Research. The Belmont Report. April 18, 1979. http://ohsr.od.nih.gov/guidelines/belmont.html.

3 US Department of Health and Human Services. Guideline for the Study and Evaluation of Gender Differences in the Clinical Evaluation of Drugs. Federal Register 58, 39406 (22 July 1993).

4 US Department of Health and Human Services, Food and Drug Administration. Final Rule on Investigational New Drug Applications: Amendment to Clinical Hold Regulations for Products Intended for Life-Threatening Diseases and Conditions. Federal Register 65, 34963-71 (1 June 2000).

5 Council for International Organizations of Medical Sciences (CIOMS) in collaboration with the World Health Organization (WHO). International Ethical Guidelines for Biomedical Research Involving Human Subjects. Geneva, November 2002. www.cioms.ch/guidelines_nov_2002_blurb.htm.

6 Kleist P. Women and Trials: When is Gender a Consideration? Applied Clinical Trials 2005; June: 72-84.

7 National Institutes of Health Revitalization Act. Public Law 103-43; 1993.
8 Harris DJ, Douglas PS. Enrolment of women in cardiovascular clinical trials funded by the National Heart, Lung, and Blood Institute. N Engl J Med 2000;343:475-80.

9 Vidaver RM, Lafleur B, Tong C, Bradshaw R, Marts SA. Women subjects in NIH-funded clinical research literature: lack of progress in both representation and analysis by sex. J Womens Health Gend Based Med 2000;9:495-503.

10 Rochon PA, Clark JP, Binns MA, Patel V, Gurwitz JH. Reporting of gender-related information in clinical trials of drug therapy for myocardial infarction. CMAJ 1998;159:321-7.

11 Lee PY, Alexander KP, Hammill BG, Pasquali SK, Peterson ED. Representation of elderly persons and women in published randomized trials of acute coronary syndromes. JAMA 2001;286: 708-13.

12 Ramasubbu K, Gurm H, Litaker D. Gender bias in clinical trials: do double standards still apply? J Womens Health Gend Based Med 2001;10:757-64.

13 Heiat A, Gross CP, Krumholz HM. Representation of the elderly, women, and minorities in heart failure clinical trials. Arch Intern Med 2002;162: 1682-8.

14 Johnson SM, Karvonen CA, Phelps CL, Nader S, Sanborn BM. Assessment of analysis by gender in the Cochrane Reviews as related to treatment of cardiovascular disease. J Womens Health 2003; 12:449-57.

15 Bandyopadhyay S, Bayer AJ, O’Mahony MS. Age and gender bias in statin trials. QJM 2001; 94:127-32.

16 The Digitalis Investigation Group. The effect of digoxin on mortality and morbidity in patients with heart failure. N Engl J Med 1997;336:525-33.

17 Rathore SS, Wang Y, Krumholz HM. Sex-based differences in the effect of digoxin for the treatment of heart failure. N Engl J Med 2002;347:1403-11.

18 Ridker PM, Cook NR, Lee I-M, Gordon D, Gaziano JM, Manson JE, et al. A randomized trial of low-dose aspirin in the primary prevention of cardiovascular disease in women. N Engl J Med 2005;352:1293-304.

19 Bundesministerium für Gesundheit und Soziale Sicherung in Deutschland. Verordnung über die Anwendung der Guten Klinischen Praxis bei der Durchführung von klinischen Prüfungen mit Arzneimitteln zur Anwendung am Menschen. 9. August 2004. Bundesgesetzblatt Teil I, Nr. 42; 2004. 